\title{
Oil and Gas Pipeline Monitoring using Artificial Neural Network
}

\author{
Nuka D. Nwiabu \\ Department of Computer Science \\ Rivers State University \\ Port Harcourt, Nigeria
}

\author{
Kingsley E. Igbudu \\ Department of Computer Science \\ Rivers State University \\ Port Harcourt, Nigeria
}

\begin{abstract}
Conventional Artificial Neural Network approaches such as Feed-Forward Networks has been used in diverse applications but are not naturally predictive and also require supervised learning. Feed-forward Artificial Neural Network also trained by backpropagation poses the problem of varnishing gradient. Long Short Term Memory is an Artificial Neural Network recurrent technique that allows long range contextual representation to be stored and learnt in an unsupervised manner. In this work a modified Sparse Distributed LSTM Algorithm using Gaussian membership function with a context-decision gate for detection and monitoring operations has been proposed as an alternative to the traditional FeedForward Architecture. The AI monitoring System shows promising results in solving many recurrent problems, particularly those requiring long-term storage dependencies the Vanishing Gradient problem (VGP) and has the ability to use contextual information when mapping between input and output sequences. The Oil and Gas AI monitoring system employs dynamic data flow modeling to simulate the behavior of probably militant behaviors. The contextual information (context data) includes such context as Pressure; Vehicle passing along the pipeline area, Manual digging, and Machine excavation. Dynamic simulations were performed using a real-time data obtained from the SPDC. The data is tested using AI system in MATLAB-SIMULINK environment to verify the performance of the proposed system. The results were promising indicating the real state of vandalism prediction.
\end{abstract}

\section{General Terms}

Surveillance System, Safety and security system

\section{Keywords}

Artificial neural network, Sparse Distributed LSTM, vandalism, Oil and Gas pipeline monitoring, Recurrent Neural Network.

\section{INTRODUCTION}

Conventional ANN approaches such as feed-forward neural networks are very popular and being widely used in diverse applications which includes classification of plant species, prediction of heart diseases, oil pipeline condition assessment [1]. However, conventional approaches used are more not naturally predictive and also require supervised labeling or learning [2]. Typically, a standard feed-forward ANN trained by back-propagation possess the problem of vanishing gradients [3]. Thus, recurrent techniques such as LSTM have been developed to attack/counteract this problem. With this solution long range contextual representations can be stored and even learnt in an unsupervised manner. However, the
LSTM still is not sufficient in its present form for decision making. In order to bridge this gap, a concept termed contextdecision gates is introduced to make LSTM decision capable in predictive systems. This main aim of this work is to exploit some pipeline contextual information to build An AI pipeline monitoring System using the sparse distributed Long-Short Term Memory to monitoring against oil and gas pipeline facilities vandalism. It is desired to red-flag as suspicious any activity across the pipeline area with high value of abnormality. The Pipeline monitoring system will be useful as a model for understanding artificial intelligent technique. It will afford one the opportunity to explore new grounds in predictive decision making system with long range contexts. The study will border on the sequence learning recurrent neural network based on Long Short Term Memory (LSTM). In particular, the system will exploit the AI system for the detection of potential threats to a pipeline using a real life dataset having similar features to situation in the pipeline installations in Niger Delta regions of Nigeria.

The remainder of the paper is as follows. The following session provides; 2. Brief overview of related works, 3.The design and research methodologies adopted are presented. 4. Predictive System using ANNLSTM 5.Confronting oil and gas Pipeline Monitorization using LSTM based Oil and Gas Pipeline Monitoring AI System, 6. Results and Discussion. Finally, a conclusion is drawn.

\section{RELATED WORKS}

The field of threat identification and risk assessment in industries is an active one with recent progress made in both understanding and application oriented approaches.

In [4], a fault diagnosis system for interdependent critical infrastructures was developed and applied to a synthetic benchmark Energy network (IEEE 30 bus model); this system used the Hidden Markov Model (HMM) - a state based model that uses time as its operational learning parameter. Such models are useful as time-observers in fault-critical situations. Using the HMM model, the quantification of cyber-paths of critical infrastructures (CIs) was attempted. The cyber-paths include BGP routing protocols, SCADA servers, corporate networks etc. The paths are built-in to a state model that varies through all possible states (ergodic HMMs). This approach used the probabilistic distance metric (PDM) for decision making and the torch framework for simulation experiments. Performance measures based on false positive and negative rates and the detection delay was shown to be encouraging. However, one drawback in using the HMM is its inability to fully account for the state space - as the capability of detecting a possible threat to a $\mathrm{CI}$ is a function of time and space. 
The use of Bow-ties - a diagrammatic cause consequencebarrier model, as a risk/threat detection and monitoring mechanism for oil and gas and similar environments have been investigated in [5]. Their review of existing bow-tie schemes from both the qualitative and quantitative bow-tie paradigm identified some competing intelligent schemes bordering on Fuzzy logic, Bayesian networks and Boolean logical calculus. Their review also identified the limitations of the bow-tie as its inability to capture comprehensively a realistic real world model to assess the risks involved in a facility or environment under threat.

Using an agent based model and regression-based statistical design of experiments (doE),[6] were able to simulate pirate behavior that accounts for exploitation of marine environments in Somalia. With the Concept of Operations (CONOPS) agent, they tried to determine which factors of the Meteorology and Oceanography conditions (METOC) likely influence pirate behavior and increase the chances of a threat to existing facilities - these factors or parameters was then allocated more intelligent resources from a mobile pirate control system. However, they found out that using the doE approach with CONOPs for the different regression models did not present a clear cut direction as to which parameters or conditions are more useful.

In [7], the potentials of a Bayesian network for effective identification of threats due to piracy to oil industry infrastructure was investigated. They identified the inadequacies of existing systems in handling multi-parameter conditions with a high degree of state variability - which are a more frequent feature such environments. In this vein, they optimized the SARGOS - a system developed by the French private and public research center for offshore warning and system response protection using the Bayesian approach. In particular, the Bayesian model was used to automate the preparation of response plans of the SARGOS. For validation purposes, they used the IMO database - a piracy and armed robbery database for constructing the Bayesian network. The knowledge in the Bayesian model was extended by industry experts in the maritime security sector and integrated into SARGOS to form the SARGOS Bayesian Network. Using this network, simulation experiments were then performed for different attack scenarios; attacks on floating, production, storage and offloading by an unknown private vessel (FPSO) with reported promising results. However, this model requires manual tuning by experts and does not support dynamic targets (i.e. moving vessels or platforms).

A context-based situation and threat assessment system for harbor surveillance was developed in [8]. The system uses a two-level architecture; a low level ontology-based model for reasoning, by deducing and classifying harbor situations and objects respectively and a high level belief-argumentation model for threat evaluation due to suspicious vessels. Violations to the norm are then checked with the assumed set of argumentation values.

\section{METHODOLOGIES}

\subsection{Action Research}

Is a collaborative progressive problem solving methodology well suited for researchers and organizational practitioners [9]. Going by the definition, for a detailed understanding, AR can be said to be a methodology that deals with "learning by doing", that is to say that individuals or a team of researchers identifies a problem, do what they can to resolve the problem, see the level of success they have made so far, and if the target is not met, they try again. In this study we strictly followed the Susan cycle (spiral) approach, since the whole purpose of action research is to determine simultaneously an understanding of the social system and the best opportunities for change in any system. The interest of this research study is to develop a predictive decision support system that will support organizational decisions in problem solving. Realizing this noble aim, collaboration with the domain practitioners was made to gain an understanding of the domain activities. It is based on the understanding of the domain and data collected in collaboration with field or domain engineer, coupled with an online benchmark dataset that the researcher will build his system. The data and the practitioners' requirement will be refine and redesign iteratively to suit the use for this work.

\subsection{Rational Unified Process}

In line with the AR which involves iterative refinement and redesigning of the practitioners requirement, we have employed an iterative object oriented design software engineering methodology known as RUP. The RUP aims at ensuring the production of high-quality software that meets the needs of its end-users, within a predictable schedule and budget [10]. RUP development team works in collaboration with the customers, partners, Rational's product groups as well as Rational's consultant organization, to ensure that the process is continuously updated and improved upon to reflect recent experiences and evolving and proven best practices. A RUP activity creates and maintains models and emphasizes the development and maintenance of models-semantically rich representations of the software system under development [11]. RUP is a guide for how to effectively use the Unified Modeling Language (UML). The UML is an industrystandard language that allows us to clearly communicate requirements, architectures and designs [12]. The UML was originally created by Rational Software, and is now maintained by the standards organization Object Management Group (OMG).

\section{PREDICTIVE SYSTEM USING ARTIFICIAL NEURAL NETWORK}

An artificial neural network can "learn" a task by repeated adjustments of weights [13]. ANN is made up of hierarchy of layers, and the neurons in the networks are arranged along these layers, these neurons connected to the external environmental form input and output layers [14]. The weights are now modified to bring the network input/output behaviour into line with that of the environment. When the neuron is supplied with the input and numerical weight, it has a means of computing its activation function and sends it as an output signal through the output link. The input signal can be raw data or outputs of other neurons. The output signal can either be the final solution to the problem or an input to other neurons. The neuron computes the weighted sum of the input signals and compares the result with a threshold value $\alpha$. If the total input is less than the threshold, the neuron output is 0 . But if the total input is greater than or equal to the threshold, the neuron becomes activated and its output attains a value 1 . Mathematical model description of the artificial neuron for simplicity is given below:

$A=\sum_{i=0}^{n} a i w i$ 
$B=\left\{\begin{array}{l}1 \text { if } A \geq \propto \\ 0 \text { if } A<\propto\end{array}\right.$

Where;

$>A$ is the total weighted input to the neuron

$>a_{i}$ is the value of the input as $i$ ranges from 0 to $\mathrm{n}$

$>\quad w i$ is the weight of input as I ranges from 0 to $\mathrm{n}$

$>\mathrm{n}$ is the number of neuron inputs

$>\quad \mathrm{B}$ is the output of the neuron

The above equation is known as the Transfer or activation, which is also called the sign function- that defines the property of the artificial neuron. This is chosen on the basis of the type of problem the artificial neuron needs to solve. Hence the actual output of the neuron with sign activation is represented in the equation (2) below;
$B=\operatorname{sign}\left[\sum \operatorname{aiw} i-\propto\right]$

\section{CONFRONTING OIL AND GAS PIPELINE MONITORIZATION USING LSTM BASED OIL AND GAS AI MONITORING SYSTEM}

The main goal of this work is to exploit the available oil and gas pipeline contextual information to provide hypothesis on suspicious activities across the pipeline area. It is desired to red-flag any activity that persist over time and that approaches a restricted value or a set threshold based on the activity been carried out at a particular time. The architecture of the proposed Pipeline Monitoring system which is a sequence learning recurrent neural network based on Long-Short Term Memory is as shown in fig. 1 .

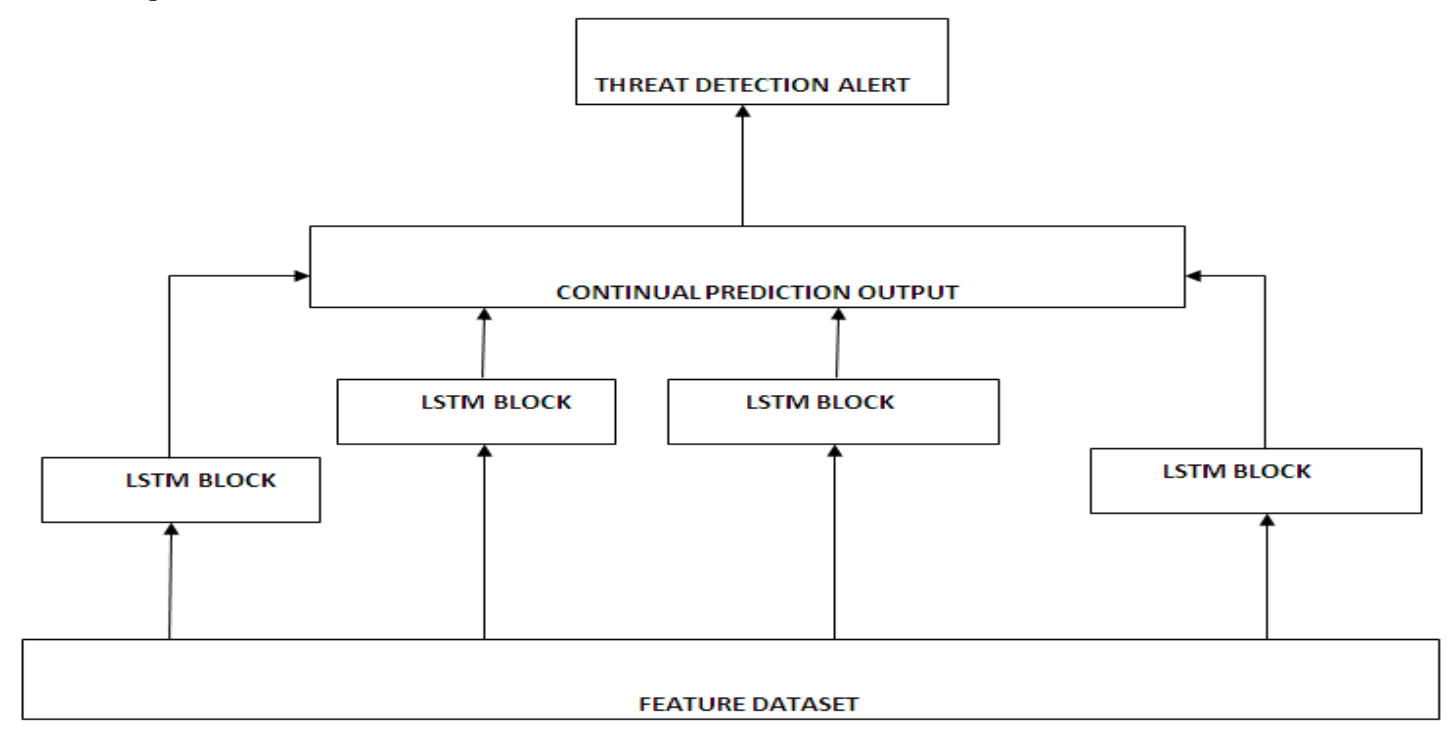

Fig 1: Proposed System Architecture

The system works as described; the contextual information (feature dataset) is broken down into a numerical context prediction activity; using the feature dataset module. The context information base on the help of sensors are fed to an LSTM block which learns a sequential representation of the context in the previous time step and then predicts the most likely sequences at the next time step. The predictions are then sent to a threat alert which flags predicted threat levels with high (abnormal) values. A detailed of the system can be made using a use case and a Concept level design use case as shown in fig. 2 and fig. 3 . Figure 2 captures a typical scenario in which wireless sensors are deployed in an unattended environment to monitor and collect data of certain threat related activities from the region of interest to an LSTM AI Monitoring system and then to the control room where it is been seen by a human security personnel. The LSTM Monitoring AI system model keeps track of these activities based on the incoming sensory information from the wireless sensors mounted at several pipelines area. If a particular activity persist over time and is inconsistent with the set value of the area of interest, the System predicts it, otherwise it ignores (forgets) it. The predicted activity is passed to a threat alert which flags a particular threat to the pipelines. 


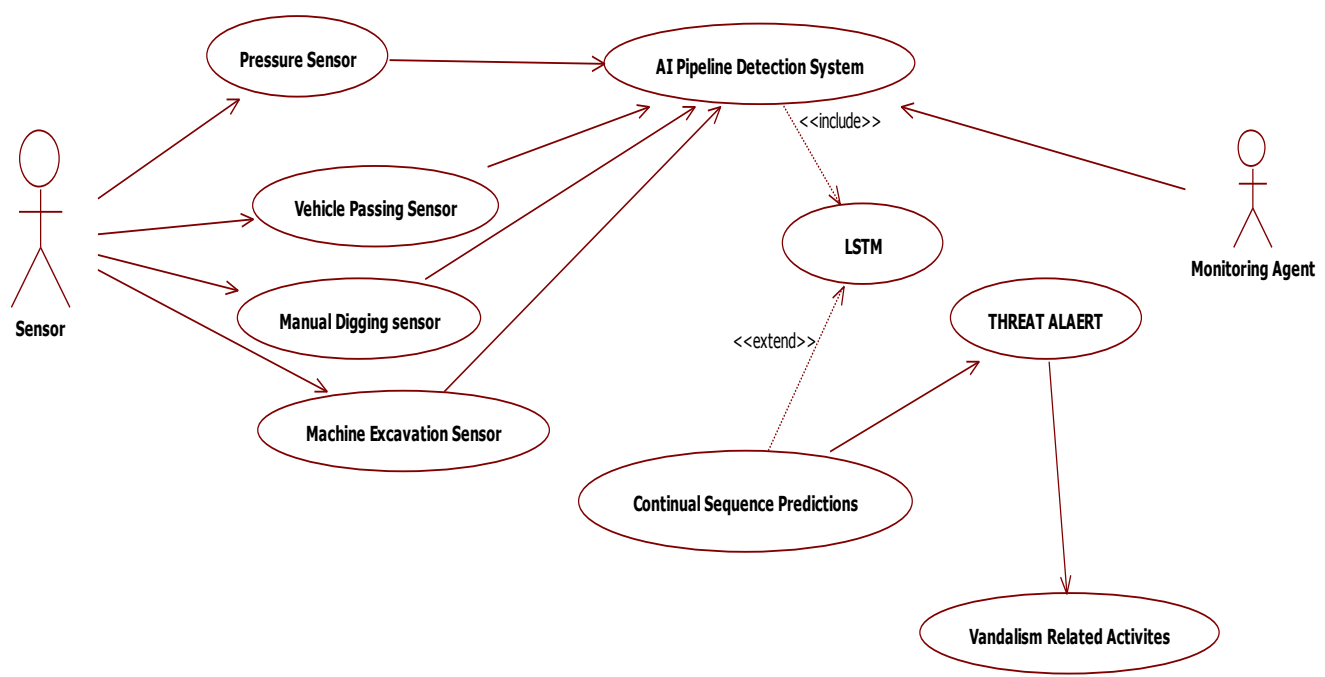

Fig 2: A Use-case model of the Proposed Pipeline Monitoring System

Fig. 3 as can be observed contains concatenated local contextual parameters which are incorporated into Pipeline Monitoring System as trafficability values using wireless sensor. The trafficability are values between zero and one, where zero indicates no threat and one indicates threat. The pipeline local contextual data includes;

$\checkmark$ Pressure

$\checkmark \quad$ Vehicle Passing

$\checkmark \quad$ Manual Digging

$\checkmark$ Machine Excavation
The sensed signals (context parameters) are fed into the context learning module(LSTM subsystem) through a multiplexer, and are transformed and seen by the LSTM as words, The LSTM subsystem keeps track of the sensed signals in the memory module and then passes these signals to the control subsystem(Prediction module), which combines the individual trafficability values corresponding to each piece of contextual information into a value that would be used to indicate situations that poses danger and the one that does not.

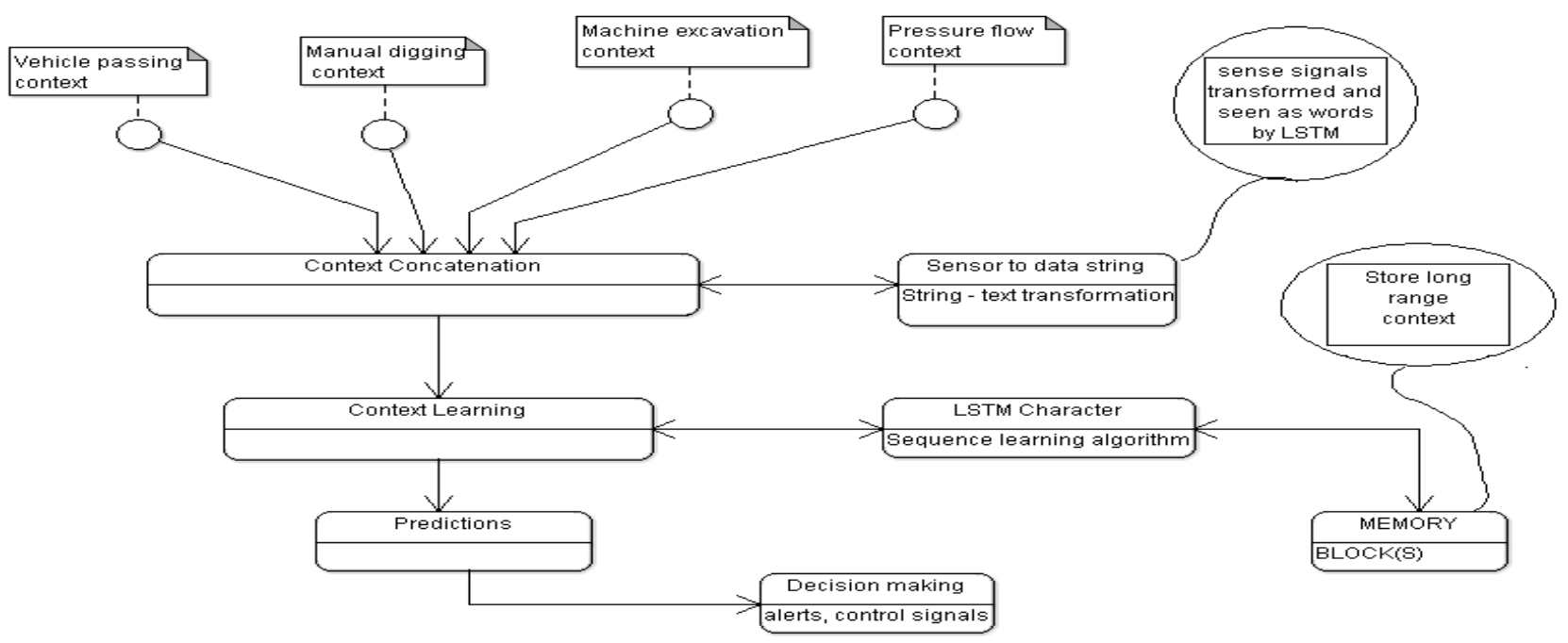

Fig 3: Concept-level situation aware design

A screenshot of the Pipeline Monitoring system based on the Long-Short Term Memory is depicted in fig.4. the PMS system uses the Gaussian membership function in simulink.

Gaussian built-in membership function Syntax;

$Y=\operatorname{gaussm} f(X,[\operatorname{sig} C])$

The symmetric gaussian function depends on two parameters $\partial$ and $\mathrm{c}$ as given by

$f(X, \partial, C)=e^{\frac{-(x-c)^{2}}{2 \partial^{2}}}$
The parameters for gaussmf represent the parameters and $\mathrm{c}$ listed in order in the vector [sig c]. Where $\mathrm{c}$ is the mean, and $\partial$ the variance.

The deployment interface includes the following parts:

Random Source: this part is made up of;

i. Predicted Parameters sensor blocks: this converts the real time parameters like pressure, vehicle passing, manual digging and machine excavation, etc. into electrical signal 
ii. Display Sensor block: displays the numerical state of the sensed data

iii. Multiplexer block; That concatenates the entire sensed signal and synchronizes them through a transmission line to the LSTM subsystem.
> LSTM Subsystem: The LSTM subsystem passes these signals through different signal line (transmission line) to the control subsystem.

$>$ Control Subsystem: the control subsystem predicts the sensor output based on the signal it received from the LSTM subsystem and displays a 1 to any predicted activity that poses threat and 0 to the activity that poses no threat.

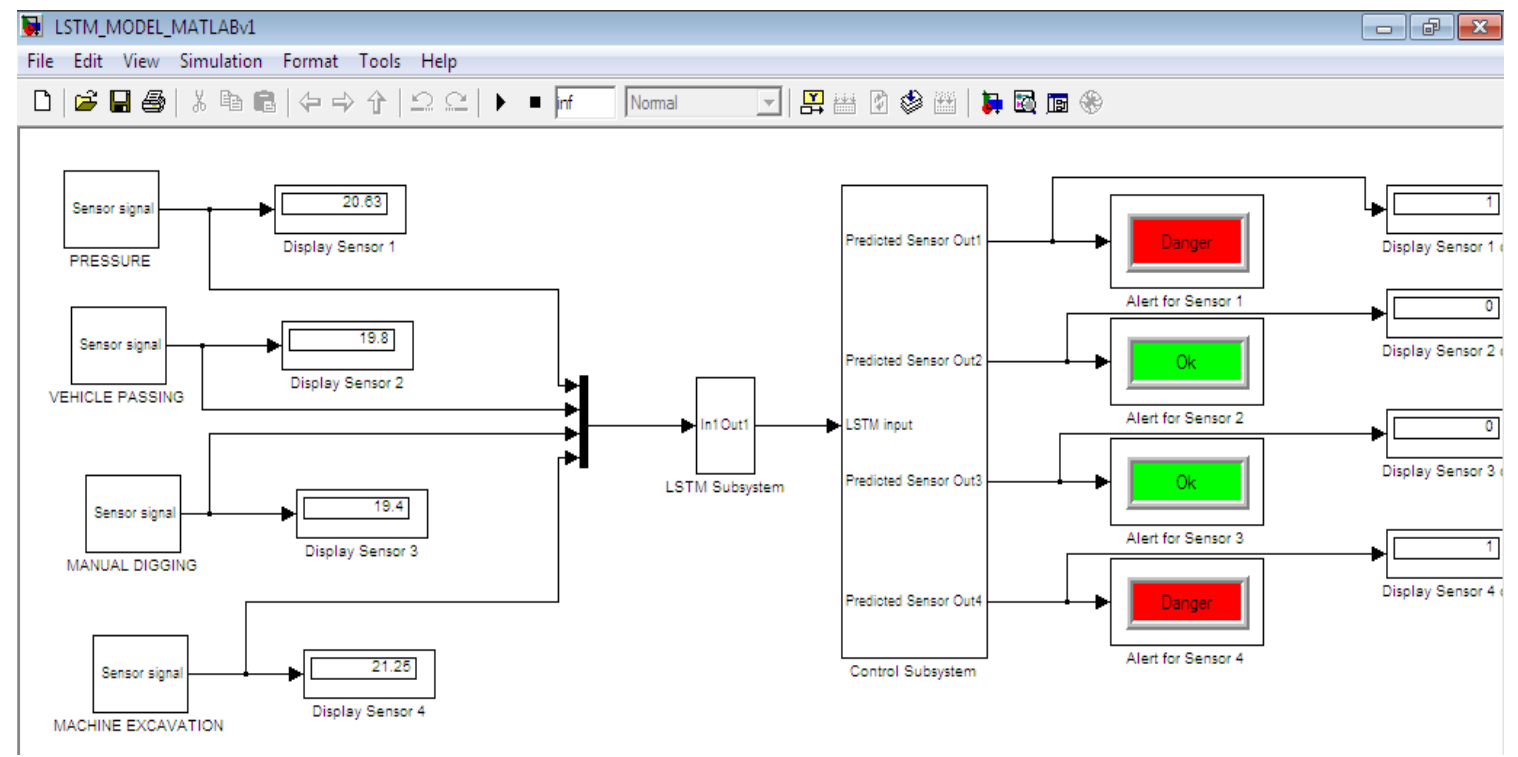

Fig 4: A screen shot of the LSTM Based Pipeline Monitoring System User Interface

\section{RESULTS AND DISCUSSION}

In order to evaluate the performance of the system, the system was tested using local pipeline contextual feature dataset from the SPDC. The area of interest is divided into four which are termed trafficability information or context data as describe in section 5. Results of tests have been tabulated in Table 1 and 2.The incorporation of trafficability data into the model allows variations in result with the given contextual information. The results shows simulation reports after several runs of AI Pipeline Monitoring system. Simulation results shows that when a particular activity is consistent with the targeted value of the trafficability data, such activity would not be red-flag, but gives a signal of green showing that the pipeline is safe. For instance, the set point for pressure drop and rising as oil flows along the pipeline is given to be $14.05 \mathrm{bar}$ and $19.99 \mathrm{bar}$. If the pressure wave drops below $14.05 \mathrm{bar}$, the system will predict danger, when the pressure wave is between $14.05 \mathrm{bar}$ and $19.99 \mathrm{bar}$, the prediction will be normal, but any rise above 19.99bar poses threat to the oil pipeline and the system predicts danger. For the vehicle passing, any weight of vehicle above $19.99 \mathrm{~kg}$ is a threat to the pipeline. A digging above the depth of 17.99inches for manual digging and machine excavation poses threat to the pipeline, the pipeline monitoring system predicts and sends signal to the control that red-flags the predicted activity as DANGER, otherwise ignores (OK).The results shows good performance of the predictive AI system for higher learning units and runs and its unique capability to make multiple predictions.
Table 1: Simulation Results

\begin{tabular}{|l|l|l|l|l|}
\hline & $\begin{array}{l}\text { Max Trial } \\
=3\end{array}$ & $\begin{array}{l}\text { Hidden Unit } \\
\text { Size = 20 }\end{array}$ \\
\hline Time(hrs) & $\begin{array}{l}\text { Pressure } \\
\text { bar) }\end{array}$ & $\begin{array}{l}\text { Vehicle } \\
\text { Passing } \\
\text { (kg) }\end{array}$ & $\begin{array}{l}\text { Manual } \\
\text { Digging } \\
\text { (inches) }\end{array}$ & $\begin{array}{l}\text { Machine } \\
\text { Excavation } \\
\text { (inches) }\end{array}$ \\
\hline $6: 00$ & 19.66 & 17.39 & 20.71 & 20.37 \\
\hline $0: 00$ & 19.43 & 21.36 & 19.81 & 19.55 \\
\hline $10: 00$ & 19.43 & 20.05 & 19.99 & 20.79 \\
\hline $22: 00$ & 18.71 & 19.99 & 19.23 & 19.85 \\
\hline $2: 00$ & 19.95 & 20.27 & 19.35 & 18.67 \\
\hline $0: 00$ & 17.71 & 18.55 & 19.82 & 20.23 \\
\hline $18: 00$ & 19.74 & 19.41 & 18.12 & 19.35 \\
\hline $16: 00$ & 18.89 & 19.85 & 21.20 & 21.01 \\
\hline $22: 00$ & 20.08 & 19.76 & 17.78 & 21.77 \\
\hline $2: 00$ & 20.57 & 19.45 & 20.19 & 20.65 \\
\hline $10: 00$ & 17.38 & 18.38 & 20.23 & 19.30 \\
\hline $4: 00$ & 20.36 & 20.94 & 20.20 & 20.30 \\
\hline & & & & \\
\hline & & & & \\
\hline
\end{tabular}




\begin{tabular}{|l|l|l|l|l|}
\hline $6: 00$ & 19.75 & 19.92 & 21.12 & 20.63 \\
\hline $8: 00$ & 20.95 & 21.98 & 19.06 & 20.76 \\
\hline $4: 00$ & 22.11 & 19.01 & 20.71 & 20.70 \\
\hline $10: 00$ & 21.83 & 20.98 & 20.92 & 19.71 \\
\hline $0: 00$ & 21.73 & 20.38 & 20.22 & 21.08 \\
\hline $16: 00$ & 20.32 & 18.49 & 21.59 & 19.89 \\
\hline $6: 00$ & 19.46 & 20.02 & 21.11 & 18.87 \\
\hline $2: 00$ & 18.73 & 19.42 & 20.44 & 18.87 \\
\hline $0: 00$ & 20.13 & 20.68 & 19.97 & 20.26 \\
\hline
\end{tabular}

Table 2: Threat Events Prediction Table Based on Simulation Result

\begin{tabular}{|l|l|l|}
\hline $\begin{array}{l}\text { Contextual } \\
\text { parameters }\end{array}$ & Sensory Signal & Label \\
\hline Pressure & 19.66 & 0 OK \\
\hline Vehicle Passing & 20.05 & 1 DANGER \\
\hline Manual Digging & 17.88 & 0 OK \\
\hline $\begin{array}{l}\text { Machine } \\
\text { Excavation }\end{array}$ & 16.78 & 0 OK \\
\hline $\begin{array}{l}\text { Pressure } \\
\text { Vehicle Passing }\end{array}$ & 19.78 & 1 DANGER \\
\hline Manual Digging & 21.65 & 0 OK \\
\hline $\begin{array}{l}\text { Machine } \\
\text { Excavation }\end{array}$ & 20.8 & 1 DANGER \\
\hline
\end{tabular}

Table 2 is a breakdown of the Predicted output of table 1 with the trafficability data and the labels. The trafficability are values between zero and one, where zero indicates no threat and one indicates threat.

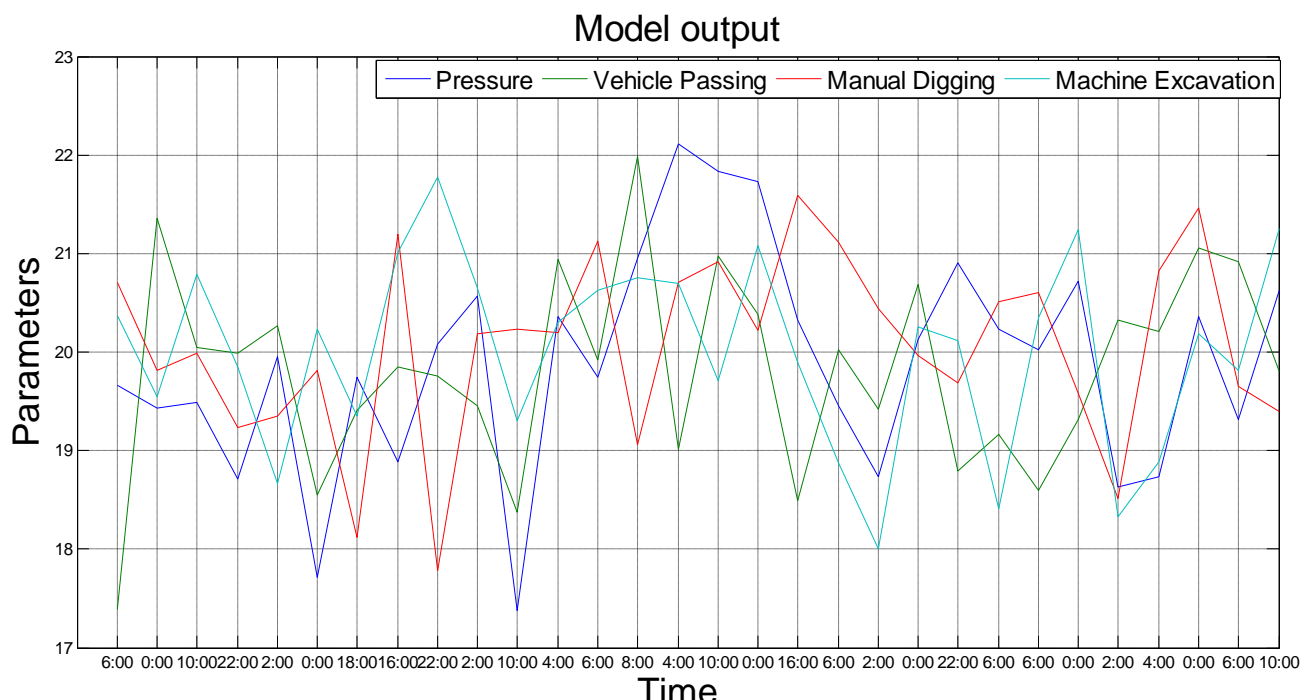

Fig.5: Graph of the Predicted Output against Time

A plot of the predicted output parameter against Time is shown in fig.5, firstly, the blue line indicates the rising and dropping of pressure wave at different time interval, at 6:00am the pressure wave is 19.66bar, at 0:00am the wave is $19.43 \mathrm{bar}$, at 10:00am the pressure dropped to 17.38 , all of which indicates no threat because they fall between the range of the set point. At $4: 00 \mathrm{pm}$ the pressure rises again to $22.11 \mathrm{bar}$ and at $22: 00 \mathrm{pm}$ it drops again to $21.71 \mathrm{bar}$ which are above rising threshold and indicates threat to the pipeline. The green line as shown in fig.5 indicates the different weight exerted on the pipeline as vehicles passing along the oil pipeline area at different time interval. At 6:00am the weight signal to the monitoring system is $17.39 \mathrm{~kg}$ which indicates no threat, at 0:00am weight signal is $21.36 \mathrm{~kg}$ and at the time interval of 8:00am the vehicle weight is $21.98 \mathrm{~kg}$, indicting a threat to the oil pipeline. The red and sky-blue lines indicators in fig. 5 shows the manual digging and the machine excavation parameters respectively, and the maximum digging or excavation depth that should be done around the oil and gas pipeline area and otherwise predicted as threat to the pipeline facility. For the manual digging at 6:00am the digging depth is 20.71inches posing threat, at $18: 00 \mathrm{pm}$ the depth is 18.12inches indicating no threat. Finally, for the machine excavation, at 6:00am the level of excavation is 20.37inches indicating threat, at $2: 00 \mathrm{pm}$ the excavation signal on the pipeline area is 18.67 inches, showing no threat to the pipeline. 


\section{CONCLUSION AND FURTHER WORK}

In conclusion, the Oil and Gas AI Pipeline Monitoring System which is based on the LSTM holds great promises as a future neural network model if properly planned. Using functional object-oriented approach, the ideas of advanced machine learning recurrent neural networks such as the one the authors have proposed here can lead to better neural models for diverse kinds of tasks. Thus, it is desirable that researchers shift from using existing simple neural network architectures to more sophisticated ones.

Further work will be on integration of the AI Pipeline Monitoring model into real time hardware, as the system has not been integrated into real time hardware so it may not be obvious if it will perform as expected and finally, the output is not symbolic i.e. cannot be interpreted as a mathematical expression yet.

\section{REFERENCES}

[1] Monica, A. and Collopy, F. 1998. Journal of Forecasting 17, 481- 495

[2] Krenker, A., Janez, B. and Kos, A. 2011. Introduction to the Artificial Neural Network - Methodological advances and Biomedical Application ISBN: 978-953-307-243-2

[3] Hochreiter, S., and Schmidhuber, J. 1997. Long shortterm memory. Neural computation, 9(8), 1735-1780.

[4] Ntalampiras, S., Soupionis, Y.,and Giannopoulos, G. 2015.A fault diagnosis system for interdependentcritical infrastructures based on HMMs.Reliability Engineering \& System Safety, 138, 73-81.
[5] de Ruijter, A., and Guldenmund, F. 2016. The bowtie method: A review. Safety Science

[6] Esher, L., Hall, S., Regnier, E., Sánchez, P. J., Hansen, J. A., and Singham, D. 2010. Simulating pirate behavior to exploit environmental information. In Simulation Conference (WSC), Proceedings of the 2010 winter pp. 1330-1335. IEEE.

[7] Bouejla, A., Chaze, X., Guarnieri, F., and Napoli, A. 2014. A Bayesian network to manage risks of maritime piracy against offshore oil fields. Safety Science, 68, 222230.

[8] Gómez-Romero, J., Serrano, M. A., García, J., Molina, J. M., and Rogova, G. 2015. Context-based multi-level information fusion for harbor surveillance. Information Fusion, 21, 173-186.

[9] Nwiabu, N.D. 2012. Situation Awareness Approach to Context-Aware Case-Based Decision Support, A thesis submitted in partial fulfillment of the requirements of Robert Gordon University for the degree of Doctor of Philosophy

[10] Jacobson, I., Grady, B., Rumbaugh, J. and AddisonWesley. 1999 Unified Software Development Process,

[11] Grady B. and Addison-Wesley. 1995. Object Solutions

[12] P. Kruchten, R. Capilla JC Duenas - The Decision view's role in software architecture practice

[13] Suzuki, K. 2011. Artificial Neural NetworksMethodological Advances and Biomedical Applications

[14] Negnevitsky, M. 2002. Artificial Intelligence: A Guide to Intelligent Systems 3rd Edition. 